\title{
Spatial variations of microbial communities in abyssal and hadal sediments across the Challenger Deep
}

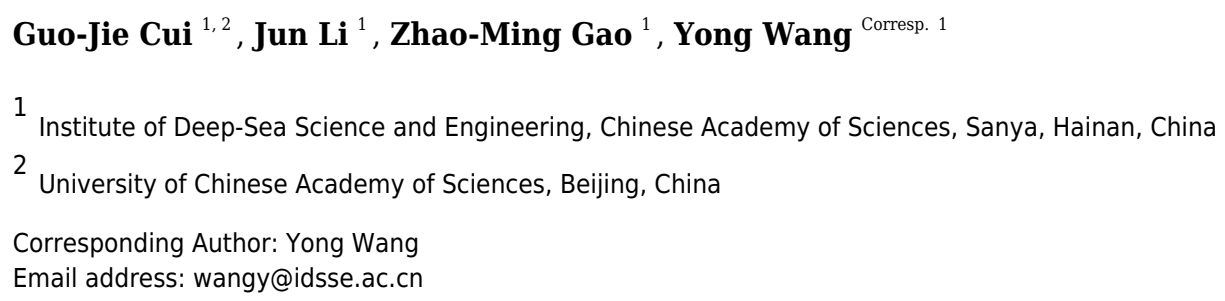

Microbial communities in hadal sediments are least explored in hadal zone $(>6,000 \mathrm{~m})$, especially in the Challenger Deep with high pressure ( 110 M pa at the bottom). In this study, we investigated the microbial communities in the sediments of the slope and trench-axis bottom of the Challenger Deep in the Mariana Trench. Classification of the reads of the $16 \mathrm{~S}$ rRNA gene amplicons showed vertical distribution of prokaryotic microbial inhabitants from the surface to up to 60 centimeter below surface floor (cmbsf). The most dominant phyla were Proteobacteria, Chloroflexi, Actinobacteria, Planctomycetes, and candidate phyla Patescibacteria and Marinimicrobia. Distinct dominant groups in the microbial communities were observed in trench-axis sediment (water depth $>8,600 \mathrm{~m}$ ), compared to the slopes of the Challenger Deep. A sampling site at the northern slope was enriched with archaea from mesophilic Euryarchaeota Marine Group II (MGII) as a biomarker of specific geochemical setting. Among archaeal community, Thaumarchaeota represented by Nitrosopumilus were dominant in the upper layers and diminished drastically in the deeper layers. "Ca. Woesearchaeota", however, became the dominant group in the deeper layers. Overall, our study provides a better understanding on the pattern of the microbial communities in the deepest hadal sediments on Earth, and highlights the extraordinary diversity still waiting to be discovered. 
$1 \quad$ Spatial variations of microbial communities in abyssal and hadal

5

7 China

$8 \quad{ }^{2}$ University of Chinese Academy of Sciences, Beijing, China

9

11 Yong Wang

12 wangy@idsse.ac.cn

13 Tel: (86) 898-88381062

14 Fax: (86) 898-88222506

15

16

17

18

19

20

21

22

23

24

25

26

27

(8)

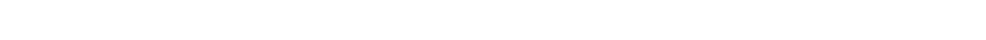

\section{sediments across the Challenger Deep}

\author{
Guo-Jie Cui ${ }^{1,2}$, Jun $\mathrm{Li}^{1}$, Zhao-Ming Gao ${ }^{1}$, Yong Wang, ${ }^{1, *}$
}

Running title: Microbial communities in hadal sediments

* Corresponding author: 
Abstract

Microbial communities in hadal sediments are least explored in hadal zone $(>6,000 \mathrm{~m})$, especially in the Challenger Deep with high pressure ( 110 M pa at the bottom). In this study, we investigated the microbial communities in the sediments of the slope and trench-axis bottom of the Challenger Deep in the Mariana Trench. Classification of the reads of the 16S rRNA gene amplicons showed vertical distribution of prokaryotic microbial inhabitants from the surface to up to 60 centimeter below surface floor (cmbsf). The most dominant phyla were Proteobacteria, Chloroflexi, Actinobacteria, Planctomycetes, and candidate phyla Patescibacteria and Marinimicrobia. Distinct was enriched with archaea from mesophilic Euryarchaeota Marine Group II (MGII) as a biomarker of specific geochemical setting. Among archaeal community, Thaumarchaeota represented by 
60

\section{Introduction}

Hadal Trenches at $>6,000-\mathrm{m}$ depth are specific ocean ecosystems, and the Challenger Deep isolated from other trenches in the Western Pacific is the deepest place on Earth (Jamieson et al. 2010). Subduction plate (seamount tunneling) steepens the forearc, causing the northern slope steeper than the southern slope. The bottom of the Challenger Deep is $11 \mathrm{~km}$ long and $1.6 \mathrm{~km}$ wide, known as slot-shaped depression. As the slope sediments reach the maximum attainment, sediments slip off the slope. Sediment transport can also be triggered by debris flows and turbidity currents (Jamieson et al. 2010). The oligotrophic hadal waters in the Challenger Deep harbored detrital matter degrading microorganisms such as Chloroflexi and candidate phylum Marinimicrobia (SAR406) (Nunoura et al. 2015). In contrast, benthic oxygen consumption rate has been in situ measured in sediment sites of three hadal trenches (Glud et al. 2013; Wenzhofer et al. 2016), indicating that the seafloor microbial carbon turnover at the trench-axis was higher than those adjacent abyssal sites probably due to the slot-shaped trench-axis trapped more particulate organic matter (POM) than previously estimated (Glud et al. 2013; Nunoura et al. 2015). Along plate collision, active hydrothermal submarine volcanoes are distributed in the Mariana arc (Baker et al. 2008). Previous studies reported a slightly elevated salinity at the water depth above $9,000 \mathrm{~m}$, indicating that the microbial communities could also be shaped by the bottom water mass density (Nunoura et al. 2015; Taira et al. 2005). Therefore, with these geochemical, geophysical and geographical parameters, unique microbial communities might have been discovered at the certain slope sites, providing opportunities to develop ecological and evolutionary theories about speciation and community assembly.

It has been speculated that hadal adapted strains of microbes were distinguished from their close relative strains dwelling in shallow marine major due to biological barriers (Gallo et al. 2015; Kato et al. 1998; Nunoura et al. 2015; Wang et al. 2019b). Dominance of hadal specific ammoniaoxidizing archaea (AOA) (Wang et al. 2019b) provides a hint to the presence of various novel microbial groups in the Challenger Deep, especially at water depth $>10,000 \mathrm{~m}$. Some eukaryotic 
87

88

microbial communities in waters from the Challenger Deep were reported recently (Guo et al. 2018; Xu et al. 2018), suggesting novelty of the eukaryotes in the Mariana Trench. Currently, most studies have focused on the microbial communities in waters rather than those in sediments in the Mariana Trench probably due to difficulties in sediment sampling in this area (Nunoura et al. 2015; Tamburini et al. 2013; Tarn et al. 2016; Tian et al. 2018; Wang et al. 2019a). At present, the prokaryotic microbial community in a trench-axis bottom site $(10,300 \mathrm{~m})$ of the Challenger Deep had been briefly investigated, and revealed that the major phyla were Chloroflexi, Bacteroidetes, Planctomycetes, "Ca. Marinimicrobia" (SAR406), Thaumarchaeota and "Ca. Woesearchaeota" (Nunoura et al. 2018). With the vertical distance of $\sim 4,000 \mathrm{~m}$ in the Challenger Deep, there must be variants in the microbial communities at different sediment sites of the slopes and trench-axis bottom and novel groups could probably be uncovered. Therefore, the spatial distribution pattern of prokaryotes microbial communities in sediments of the Challenger Deep across different hadal depths and sites is still illusive to date.

Aiming to reveal the spatial variations of prokaryotic microbes and find potentially unique groups in the hadal sediments, we investigated five sediment cores from the trench-axis of the Challenger Deep and nine slope sediment cores ranging in depth from 5,480 m-7,850 m during three cruises in 2016-2017. As the first large-scale study of the microbial communities in sediments of the Challenger Deep, our results deepen our understanding on the formation of unique ecosystem in the hadal zone.

\section{Materials \& methods}

\section{Sample collection}

Fourteen sediment samples were successfully collected from the Challenger Deep in three cruises R/V Dayang 37-II (DY37II), Tansuo01 (TS01) and Tansuo03 (TS03) during June-July of 2016, June-August of 2016 and January-March of 2017 (Fig. 1). Our sediment sampling was carried out on the slopes and the trench-axis bottom sites of the Challenger Deep from abyssal to hadal depths 
114 by 'Jiaolong' manned submersible, a box sampler and a hadal lander (Fig. 1 and Table S1). All

115 these sediment cores were sliced into 2-cm subsamples except for three cores (T1L10, T3L08 and

116 T3L11 above 10,000 m) that were sectioned into 3-cm subsamples. The ion concentrations of two

117 sediment cores were measured with an Ion chromatography system (Dionex Corporation,

118 Sunnyvale, CA, USA). All subsamples for community analysis were preserved at $-80{ }^{\circ} \mathrm{C}$ until

119 DNA extraction. The research has been permitted and secured from the Federated States of

120 Micronesia.

121

122

DNA extraction and Quantitative PCR (qPCR) analyses

123

124

125

126

127

128

129

130

131

132

133

134

135

136

137

138

139

140

Genomic DNA was extracted from $2 \mathrm{~g}$ of sediment subsamples using the PowerSoil DNA Isolation Kit (MO BIO Laboratories, Inc., Carlsbad, USA). The quality and quantity of the genomic DNA were checked with a NanoDrop spectrophotometer (ND-1000, Nanodrop Technologies, Wilmington, DE, USA) and gel electrophoresis. The abundance of 16S rRNA was quantified as an average of three replicate analyses. The prokaryotic SSU rRNA gene was quantified using the primer Uni516F/Uni806R using the StepOnePlus ${ }^{\mathrm{TM}}$ Real-Time PCR System (Applied Biosystems, Foster City, USA). The primer sequences and qPCR conditions were as described previously (Nunoura et al. 2018).

\section{PCR amplification of the $16 \mathrm{~S}$ rRNA genes}

The 16S rRNA genes were amplified with a pair of universal primers: $341 \mathrm{~F}$ (5'CCTAYGGGRBGCASCAG-3') (Zakrzewski et al. 2012) with a tagged six-nucleotide(nt) barcode and reverse primer 802R (5'-TACNVGGGTATCTAATCC-3') (Nossa et al. 2010) that target V3-V4 variable regions. The PCR reaction was prepared according to the PrimerSTAR ${ }^{\circ}$ HS DNA Polymerase (TaKaRa, Dalian, China) with $2 \mu$ of forward and reverse primers $(10 \mu \mathrm{M})$ and $2 \mathrm{ng}$ of template DNA. The PCR was performed on a thermal cycler (Bio-Rad, USA) in the following thermal cycles: $98^{\circ} \mathrm{C}$ for $10 \mathrm{~s}, 26$ cycles of $98^{\circ} \mathrm{C}$ for $10 \mathrm{~s}, 50^{\circ} \mathrm{C}$ for $15 \mathrm{~s}, 72^{\circ} \mathrm{C}$ for $30 \mathrm{~s}$, and a final extension at $72^{\circ} \mathrm{C}$ for 5 min. PCR products were purified using the TaKaRa Agarose 
141 Gel DNA Purification Kit (TaKaRa, Dalian, China) and Qubit 2.0 Fluorometer (Invitrogen, 142 Carlsbad, CA, USA) for quantification.

143

144

145

146

147

\section{Analysis of the barcoded amplicons and taxonomic assignment}

An equal amount of PCR products from different samples was mixed together and subjected to sequencing on the Illumina Miseq platform $(2 \times 300 \mathrm{bp})$ in accordance with the manufacturer's recommendation. The sequencing data were processed and analyzed using QIIME version 1.9.1 (Caporaso et al. 2010b). After qualification, the remaining reads were assigned to operational taxonomic units (OTUs) at 97\% similarity level by UCLUST (Edgar 2010). Chimeric reads were identified and excluded using ChimeraSlayer (Haas et al. 2011) and singleton OTUs with one sequence were removed. For the remaining OTUs, the most abundant read of each OTU was selected as a representative for subsequent taxonomic classification. Taxonomic assignment was conducted using the Ribosomal Database Project (RDP) classifier (version 2.2) (Wang et al. 2007) by referring to the SILVA132 database with a confidence level of $80 \%$. The remaining representatives were aligned by PyNAST (Caporaso et al. 2010a) and a phylogenetic tree was built using FastTree (Price et al. 2009). Subsequently, alpha diversity was calculated with three parameters: observed OTUs, Chao1, and Shannon Index. Using percentages of the genera in the microbial communities, Bray-Curtis dissimilarity between the layers was calculated and used for Principal coordinate analysis (PCoA) with the OmicShare tools, a free online platform for data analysis (www.omicshare.com/tools).

\section{Phylogenetic analysis of 16S rRNA genes}

The representative reads of the most abundant OTUs (Dataset1.fasta) were selected for construction of phylogenetic trees with reference sequences from the Silva and NCBI database. All the sequences were aligned using MAFFT 7.31 (Katoh \& Standley 2013) and the maximumlikelihood (ML) phylogenetic trees were constructed using RaxML (Silvestro \& Michalak 2012) with GTRGAMMA model. The bootstrap values were calculated based on 1000 replications. The 
168

169

170

171

172

phylogenetic outputs were visualized and edited in iTOL (Letunic \& Bork 2016).

\section{Results}

\section{Microbial abundance}

In the qPCR analysis, the abundance of the entire prokaryotic SSU rRNA gene ranged between $1.5 \times 10^{5}$ and $5.5 \times 10^{8}$ copies $\mathrm{g}^{-1}$ sediment from sediment surface to $66 \mathrm{cmbsf}$. The results showed that, with a few exceptions, microbial abundance generally decreased with increasing depth of sediment layers. The SSU rRNA gene copy numbers of the trench-axis at the surface layer (0-2 cmbsf) were observed at least an order of magnitude higher than those of the slopes (Fig. S1). Moreover, the abundance of the entire prokaryotic SSU rRNA at the trench-axis shown in this study was higher than the result reported in a recently work(Nunoura et al. 2018).

\section{Composition, diversity and species richness of microbial communities}

After quality control and filtration of chimeras and singletons, a total of 624,821 reads of $16 \mathrm{~S}$ rRNA gene amplicons for 95 sediment layers were obtained (Table S2). The rarefaction of the qualified reads resulted in the minimum 1143 sequences per sample (Table S2). All the curves did not reach the plateau (Fig. S2), indicating that more species might be discovered with more sequencing reads. The qualified reads were grouped into 123,955 OTUs and were then classified into 69 phyla. The 11 most dominant bacterial phyla (>1\%) were Proteobacteria, Chloroflexi, Actinobacteria, Planctomycetes, candidate phylum Patescibacteria, candidate phylum Marinimicrobia, Gemmatimonadetes, Bacteroidetes, Firmicutes, Acidobacteria and candidate phylum Zixibacteria (Fig. 2). The most abundant Proteobacteria in hadal sediment samples was Gammaproteobacteria composed of Pseudoalteromonas (similarity 100\% to NR_152003), Halomonas (similarity 100\% to NR_043299), Pseudomonas (similarity 100\% to NR_159318) and Alteromonas (similarity 100\% to NR_148755). These taxa varied in their percentages spanning 0\%63\% across all sediments layers. T1B08 was particularly enriched with Gammaproteobacteria highly represented by Alteromonas. Planctomycetes and Thaumarchaeota were significantly 
195

196

197

198

199

200

201

202

203

204

205

206

207

208

209

210

211

212

213

214

215

216

217

218

219

220

221

negatively correlated in their relative abundances (Correlation test with 95 samples; $P<0.00001$ ). Two archaeal phyla were abundant in the samples: Thaumarchaeota and Nanoarchaeaeota. Nitrosopumilus of Thaumarchaeota was the major group in the Challenger Deep sediment layers, while the latter represented by " $\mathrm{Ca}$. Woesearchaeota" was abundantly detected in deep layers of the deepest sediments $(>10,000 \mathrm{~m})$. The high abundance of Euryarchaeota archaea was exclusively shown in the layers of the T1B08 sample (Fig. S3).

The observed OTUs, Chaol and Shannon index were normalized based on the minimal number of reads (Table. S2). The sediments from $<6,000-\mathrm{m}$ depths were associated with the highest Chao 1 and Shannon index such as in DMC02, DD114 and DD121. The diversity indexes for the different layers in the T1B08 were the lowest among the sediments. The communities in the bottom sediments $>10,000 \mathrm{~m}$ depths were not more diversified than those in the slopes except for the T1B08. Moreover, from the surface to the bottom layers, the biodiversity indexes did not vary notably for all the samples.

\section{Clustering relationships of microbial communities}

A PCoA plotting could separate the microbial communities into two groups (the surface $0-2 \mathrm{cmbsf}$ layer samples were shown, simple IDs refer to Table S3), one composing of the slope samples $(5,400 \mathrm{~m}-7,800 \mathrm{~m})$ and another containing only trench-axis bottom samples $(8,600 \mathrm{~m}-11,000 \mathrm{~m})$.

In the former group, the samples from the northern slope could be further split from those from the southern slope. The T1B08 sample was far from the two groups in the plot (Fig. 3A), reflecting its distinct composition of microbial community. Principally, the microbial communities were clustered based on the geographic locations and depths. The combination of PCO1 and PCO2 explained $53 \%$ of the differences among the communities (Fig. 3A). Hierarchical clustering of the samples using the percentages of genera in the corresponding communities also demonstrated that the microbial communities in trench-axis sediments differed from those in the slopes (Fig. 3B). The similarity of the microbial communities was determined by sampling sites, rather than depths 
222 to the surface layers, since the communities from the same cores tend to be grouped together (Fig. 223 S4).

\section{Phylogenetic analysis of $16 S$ rRNA genes}

226

227

228

229

230

231

232

233

234

235

236

237

238

239

240

241

242

243

244

245

246

247

248

The most abundant OTUs (DatasetS1.fasta) were used to determine their phylogenetic positions. The representative reads for a total of 35 OTUs were selected for the construction phylogenetic tree with their closest relatives in the NCBI. The most abundant one was within the same group with a neighbor of Nitrosopumilus (Fig. 4A). The Nitrosopumilus in the Challenger Deep sediments resembled those inhabiting other abyssal and hadal waters and sediments from the Ogasawara Trench, the Japan Trench, and even the Puerto Rico Trench, with respect to their short phylogenetic distance. This has been observed and discussed in a recent work on the high homogeneity of Nitrosopumilus AOA in hadal zones (Wang et al. 2019b). The phylogenetic position of OTU100704 particularly enriched in T1B08 showed high affinity (100\% identity) to mesophilic archaea of Marine Group II (MGII) (estimated optimum growth temperature: $T_{\text {opt: }}=40.76$ (Kimura et al. 2013)) isolated from the Juan de Fuca hydrothermal flume (Rika E et al. 2013). The five OTUs affiliated with Nanoarchaeaeota are $C a$. Woesearchaeota widely distributed in the Challenger Deep sediments from 5,481 m to 10,953 m. Similar sequences were also obtained from saline water. The five OTUs in our results can split into 2 groups, and thus there would be more potentially novel species in this phylum.

Actinobacteria, Cyanobacteria, Firmicutes and Chloroflexi as members of superphylum Terrabacteria are almost ubiquitous in the sediments. The six OTUs of Chloroflexi in ML phylogenetic tree were split into four major groups (Fig. 4B), in which OTU124775 seems to represent a novel taxon. Interestingly, OTU344095 was approximate to Dehalococcoides spp., a known genus of strictly anaerobic bacteria with capacity of gaining energy from the reduction of chlorinated compounds (Tas et al. 2010). Furthermore, "Ca. Actinomarinales", described as a new group of photoheterotrophic Actinobacteria with ubiquitous presence in the pelagic layer of the 
249

250

251

252

253

254

255

256

257

258

259

260

261

262

263

264

265

266

267

268

269

270

271

272

273

274

275

oligotrophic ocean (Ghai et al. 2013), was also found in sediment layers especially in surface layer of T1B08. Furthermore, a proportion of 16S rRNA OTUs in the surface layer of T1B08 belonged to the picophytoplankton Synechococcus (3\%) and Prochlorococcus (7\%) in Cyanobacteria phylum (Fig. 4B). The two Cyanobacteria genera and " $\mathrm{Ca}$. Actinomarinales" originally present in the euphotic layer were rarely detected in the other sediment samples. The abundance of these euphotic bacteria in T1B08 surface layer was probably buried relicts from depositional material (Kirkpatrick et al. 2016) or might be derived from contamination during the operation of the boxcore sampler.

FCB is another superphylum mainly containing Chlorobi, candidate phylum Marinimicrobia, and Bacteroidetes (Bertagnolli et al. 2017; Getz et al. 2018; Hug et al. 2016). Nowadays, more new taxa within the FCB superphylum were recovered from sediment metagenomes (Castelle et al. 2013; Parks et al. 2017; Rinke et al. 2013), indicating their prevalence in various environments on Earth. In this study, OTU64116 was grouped with references of a newly defined phylum Zixibacteria (Castelle et al. 2013). Some of the Marinimicrobia OTUs were grouped with the sequences from previous studies, while the others represented novel groups that might have evolved in the hadal sediments (Fig. 4C).

\section{Discussion}

In this study, we revealed spatial variations of the microbial communities in the sediments from the slopes and the trench-axis bottom of the Challenger Deep. Here, we also figure out that hadal communities within the trench-axis are distinct from the hadal slopes by their major taxa and the potentially unidentified OTUs, such as some groups in Chloroflexi and candidate phylum Marinimicrobia. It is believed that the organic matter accumulation rate in sub-sea floor sediment attenuated with the increase of water depth, however, hadal trenches do not comply with this rule despite oligotrophic water column above (Luo et al. 2017). Recently, benthic oxygen consumption rate has been successfully in situ measured (Glud et al. 2013; Luo et al. 2018; Wenzhofer et al. 
276

277

278

279

280

281

282

283

284

285

286

287

288

289

290

291

292

293

294

295

296

297

2016), indicating that the seafloor oxygen consumption rates and the microbial carbon turnover at the trench-axis were higher than those adjacent abyssal sites. Furthermore, the funneling effect and erratic downslope sediment transport within the hadal trenches lead to fresher and more labile organic matter concentrated at the trench-axis bottom based on sedimentation rate that was higher than global average, together with total organic carbon contents increasing with water depth (Glud et al. 2013; Turnewitsch et al. 2014). Therefore, hadal trench-axis area is a natural vector to capture organic matter, which ultimately contributes to the different microbial communities compared to those in the slopes.

Recently, a bottom sediment core at depth of 10,300 m was investigated and geochemical data were provided by Nunoura et al. (2018). Our measurements of nitrate and sulfate in two cores (Table S4) from the trench-axis bottom were consistent with their results, but the validity of the data was questioned majorly due to decreased hydrostatic pressure. Therefore, the bottleneck of the geochemical work at such depths is to make sensors for the in situ work (Glud et al. 2013; Luo et al. 2018). To date, only trench-axis bottom oxygen consumption rate was in situ measurement at that hadal depth. In this study, we did not conduct chemical and nutrient analyses on all the sediment samples, but the dominant species resembling currently known ones could provide some hints to the local environments. Strikingly, the phylogenetic tree exhibited that most of the dominant species were grouped with known species, suggesting that these species were widespread in the trench sediments, and even in trench waters. The SAR202 bacteria as the most abundant group in Chloroflexi were degraders of various detrital materials in the deep-sea zones (Landry et al. 2017). We also revealed that potential novel group of Chloroflexi dwelling in the Challenger Deep. The genomics analysis of the Chloroflexi in hadal waters coincides with the previous studies on its recycling capacity (Landry et al. 2017; Mehrshad et al. 2017). The groups from candidate phylum Marinimicrobia were also abundant with their ability to recycle peptides and nitrogen compounds (Hawley et al. 2017). Furthermore, trenches are the habitat to specific microbial communities with specificities for pressure adaption such as the known piezophilic genera 
303

304

305

306

307

308

309

310

311

312

313

314

315

316

317

318

319

320

321

322

323

324

325

326

327

328

329

Colwellia, Shewanella, Moritella, and Psychromonas (Bartlett 1992). In this study, these major piezophilic groups of Gammaproteobacteria have already been found in our samples as well as in other hadal surface sediments (DeLong et al. 1997; Kato et al. 1998; Nogi et al. 2004; Xu et al. 2003; Zhang et al. 2018), indicating its important ecological role in hadal sediments for detrital carbon recycling. The dominant species in Planctomycetes was " $\mathrm{Ca}$. Scalindua", which is capable to reduce nitrite with ammonia in anoxic condition (Schmid et al. 2003; Woebken et al. 2008). Nitrosopumilus as an alternative ammonia oxidizer is a typically aerobic since the ammonia oxidization process under the archaea requires oxygen. A recent study showed a quick descent of $\mathrm{O}_{2}$ concentration in a surface sediment sample $(10,817 \mathrm{~m})$ (Glud et al. 2013). This explains the negative correlation in relative abundance between Planctomycetes and Thaumarchaeota in our samples.

In this study, a large number of the sediment OTUs were categorized as Nanoarchaeaeota (Woesearchaeota) phylum. Previous researchers revealed that AR20 was one of the most widely distributed "Ca. Woesearchaeota" (Castelle et al. 2015). With the first complete genome, AR20 genome was only $0.8 \mathrm{Mb}$ with incomplete core pathways, suggesting a potential symbiotic or parasitic lifestyle (Castelle et al. 2015). Additionally, the " $\mathrm{Ca}$. Woesearchaeota" found in the hadal sediments also preferred the deeper layers (Nunoura et al. 2018), suggesting that oxygen was the determining factor that affects its distribution. Moreover, Nanoarchaeaeota (Woesearchaeota) could split into at least two groups, suggesting that there should be more unidentified groups in the phylum and worthwhile to be further explored in the hadal zone. In some layers of the T1B08, MGII was one of the dominant groups. As active hydrothermal submarine volcanoes were widely distributed in the Mariana arc (Baker et al. 2008), it should not be surprising to find mesophilic archaea of MGII in T1B08 as a result of hypothetical hydrothermal process occurring in the sediments. Hence, there are more microenvironments located on the northern slope for the formation of unique but sporadic microbial communities. 
330 For the potential unidentified groups detected in this study, their roles remain unknown. Perhaps, 331 future omics work may provide their taxonomic positions and metabolic features. In addition, the 332 hadal trenches have their own characteristics. For example, the Japan Trench situated in the 333 Kuroshio Current province is relatively eutrophic (Jamieson et al. 2009; Nakatsuka et al. 1997) 334 and the South Sandwich Trench is the only sub-zero hadal zone (Vanhove et al. 2004). The 335 hypothesis of the microbial inhabitants as the markers to the sedimentary process and in situ 336 environments is perhaps not applicable to the other trenches.

337

338

339

340

341

342

343

344

345

346

347

348

349

350

351

352

353

354

355

356

The rarefaction curves and estimates of Chaol and Shannon were indicative of insufficient sequencing depth for recovery of the microbial communities in our sediment samples. To date, sequencing of 16S rRNA gene amplicons has been widely applied to study microbial communities in various environmental conditions. Selection of proper primers is one of the important factors for detection of a microbial community with high fidelity. In this study, the $341 \mathrm{~F}$ and $802 \mathrm{R}$ primers for V3-V4 region were evaluated to recover $86.4 \%$ of Bacteria and $78.7 \%$ of Archaea in the Silva database although they have been considered as the best universal primer pair (Lu et al. 2015). However, it is worth noting that the surveys based on amplification of 16S rRNA genes are always imperfect. Sampling time and variable geographic differences may also affect the detection of certain taxa.

\section{Conclusions}

Our analysis first provides spatial distribution pattern of prokaryotes microbial communities in sediment of the Challenger Deep. We also figure out the communities within the trench-axis bottom are distinct from the hadal slopes, and special microbial communities were presented at certain sites. Furthermore, some novel groups were identified based on phylogenetic trees. Overall, our results deepen our understanding on microbial ecosystem in the hadal zone.

\section{Acknowledgements}


357 We are grateful to the team members aboard the $R / V$ Tansuo01 and Tansuo03 for their safe

358 navigation and their invaluable efforts in the sampling cruises. Great thanks are given to J. Chen,

359 Y. Z. Xin and D.S. Cai for their help in sampling.

\section{Reference:}

Baker ET, Embley RW, Walker SL, Resing JA, Lupton JE, Nakamura K-i, de Ronde CEJ, and Massoth GJ. 2008. Hydrothermal activity and volcano distribution along the Mariana arc. Journal of Geophysical Research-Solid Earth 113. 10.1029/2007jb005423

Bartlett DH. 1992. Microbial life at high pressures. Science progress 76:479-496.

Bertagnolli AD, Padilla CC, Glass JB, Thamdrup B, and Stewart FJ. 2017. Metabolic potential and in situ activity of marine Marinimicrobia bacteria in an anoxic water column. Environmental Microbiology 19:4392-4416. 10.1111/1462-2920.13879

Caporaso JG, Bittinger K, Bushman FD, DeSantis TZ, Andersen GL, and Knight R. 2010a. PyNAST: a flexible tool for aligning sequences to a template alignment. Bioinformatics 26:266-267. 10.1093/bioinformatics/btp636

Caporaso JG, Kuczynski J, Stombaugh J, Bittinger K, Bushman FD, Costello EK, Fierer N, Pena AG, Goodrich JK, Gordon JI, Huttley GA, Kelley ST, Knights D, Koenig JE, Ley RE, Lozupone CA, McDonald D, Muegge BD, Pirrung M, Reeder J, Sevinsky JR, Tumbaugh PJ, Walters WA, Widmann J, Yatsunenko T, Zaneveld J, and Knight R. 2010b. QIIME allows analysis of high-throughput community sequencing data. Nature Methods 7:335-336. 10.1038/nmeth.f.303

Castelle CJ, Hug LA, Wrighton KC, Thomas BC, Williams KH, Wu D, Tringe SG, Singer SW, Eisen JA, and Banfield JF. 2013. Extraordinary phylogenetic diversity and metabolic versatility in aquifer sediment. Nature Communications 4:2120. 10.1038/ncomms3120

Castelle CJ, Wrighton KC, Thomas BC, Hug LA, Brown CT, Wilkins MJ, Frischkorn KR, Tringe SG, Singh A, Markillie LM, Taylor RC, Williams KH, and Banfield JF. 2015. Genomic expansion of domain archaea highlights roles for organisms from new phyla in anaerobic carbon cycling. Current Biology 25:690-701. 10.1016/j.cub.2015.01.014

DeLong EF, Franks DG, and Yayanos AA. 1997. Evolutionary relationships of cultivated psychrophilic and barophilic deep-sea bacteria. Applied and Environmental Microbiology 63:2105-2108.

Edgar RC. 2010. Search and clustering orders of magnitude faster than BLAST. Bioinformatics 26:2460-2461. 10.1093/bioinformatics/btq461

Gallo ND, Cameron J, Hardy K, Fryer P, Bartlett DH, and Levin LA. 2015. Submersible-and lander-observed community patterns in the Mariana and New Britain trenches: influence of productivity and depth on epibenthic and scavenging communities. Deep Sea Research Part I: Oceanographic Research Papers 99:119-133. 10.1016/j.dsr.2014.12.012

Getz EW, Tithi SS, Zhang L, and Aylward FO. 2018. Parallel evolution of genome streamlining and cellular bioenergetics across the marine radiation of a bacterial phylum. Mbio 9:e01089-01018. 10.1128/mBio.01089-18

Ghai R, Mizuno CM, Picazo A, Camacho A, and Rodriguez-Valera F. 2013. Metagenomics uncovers a new group of low GC and ultra-small marine Actinobacteria. Scientific Reports 3. 10.1038/srep02471

Peer) reviewing PDF | (2019:02:34810:2:0:NEW 7 Apr 2019) 
Glud RN, Wenzhoefer F, Middelboe M, Oguri K, Turnewitsch R, Canfield DE, and Kitazato H. 2013. High rates of microbial carbon turnover in sediments in the deepest oceanic trench on Earth. Nature Geoscience 6:284288. 10.1038/ngeo1773

Guo R, Liang Y, Xing Y, Wang L, Mou S, Cao C, Xie R, Zhang C, Tian J, and Zhang Y. 2018. Insight into the pico- and nano-phytoplankton communities in the deepest biosphere, the Mariana Trench. Frontiers in Microbiology 9. 10.3389/fmicb.2018.02289

Haas BJ, Gevers D, Earl AM, Feldgarden M, Ward DV, Giannoukos G, Ciulla D, Tabbaa D, Highlander SK, Sodergren E, Methe B, DeSantis TZ, Petrosino JF, Knight R, Birren BW, and Human Microbiome C. 2011. Chimeric $16 \mathrm{~S}$ rRNA sequence formation and detection in Sanger and 454-pyrosequenced PCR amplicons. Genome Research 21:494-504. 10.1101/gr.112730.110

Hawley AK, Nobu MK, Wright JJ, Durno WE, Morgan-Lang C, Sage B, Schwientek P, Swan BK, Rinke C, Torres-Beltran M, Mewis K, Liu W-T, Stepanauskas R, Woyke T, and Hallam SJ. 2017. Diverse Marinimicrobia bacteria may mediate coupled biogeochemical cycles along eco-thermodynamic gradients. Nature Communications 8:1507. 10.1038/s41467-017-01376-9

Hug LA, Baker BJ, Anantharaman K, Brown CT, Probst AJ, Castelle CJ, Butterfield CN, Hernsdorf AW, Amano Y, Ise K, Suzuki Y, Dudek N, Relman DA, Finstad KM, Amundson R, Thomas BC, and Banfield JF. 2016. A new view of the tree of life. Nature Microbiology 1. 10.1038/nmicrobiol.2016.48

Jamieson AJ, Fujii T, Mayor DJ, Solan M, and Priede IG. 2010. Hadal trenches: the ecology of the deepest places on Earth. Trends in Ecology \& Evolution 25:190-197. 10.1016/j.tree.2009.09.009

Jamieson AJ, Fujii T, Solan M, Matsumoto AK, Bagley PM, and Priede IG. 2009. Liparid and macrourid fishes of the hadal zone: in situ observations of activity and feeding behaviour. Proceedings of the Royal Society BBiological Sciences 276:1037-1045. 10.1098/rspb.2008.1670

Kato C, Li L, Nogi Y, Nakamura Y, Tamaoka J, and Horikoshi K. 1998. Extremely barophilic bacteria isolated from the Mariana Trench, Challenger Deep, at a depth of 11,000 meters. Applied and Environmental Microbiology 64:1510-1513.

Katoh K, and Standley DM. 2013. MAFFT multiple sequence alignment software version 7: improvements in performance and usability. Molecular Biology and Evolution 30:772-780. 10.1093/molbev/mst010

Kimura H, Mori K, Yamanaka T, and Ishibashi J-I. 2013. Growth temperatures of archaeal communities can be estimated from the guanine-plus-cytosine contents of 16S rRNA gene fragments. Environmental Microbiology Reports 5:468-474. 10.1111/1758-2229.12035

Kirkpatrick JB, Walsh EA, and D'Hondt S. 2016. Fossil DNA persistence and decay in marine sediment over hundredthousand-year to million-year time scales. Geology 44:615-618. 10.1130/g37933.1

Landry Z, Swan BK, Herndl GJ, Stepanauskas R, and Giovannoni SJ. 2017. SAR202 genomes from the dark ocean predict pathways for the oxidation of recalcitrant dissolved organic matter. Mbio 8. 10.1128/mBio.0041317

Letunic I, and Bork P. 2016. Interactive tree of life (iTOL) V3: an online tool for the display and annotation of phylogenetic and other trees. Nucleic Acids Research 44:W242-W245. 10.1093/nar/gkw290

Lu Y-Z, Ding Z-W, Ding J, Fu L, and Zeng RJ. 2015. Design and evaluation of universal 16S rRNA gene primers for highthroughput sequencing to simultaneously detect DAMO microbes and anammox bacteria. Water Research 87:385-394.

Luo M, Gieskes J, Chen L, Shi X, and Chen D. 2017. Provenances, distribution, and accumulation of organic matter in 
437

438

439

440

441

442

443

444

445

446

447

448

449

450

451

452

453

454

455

456

457

458

459

460

461

462

463

464

465

466

467

468

469

470

471

472

473

474

475

476

477

the southern Mariana Trench rim and slope: Implication for carbon cycle and burial in hadal trenches. Marine Geology 386:98-106. 10.1016/j.margeo.2017.02.012

Luo M, Glud RN, Pan B, Wenzhoefer F, Xu Y, Lin G, and Chen D. 2018. Benthic carbon mineralization in hadal trenches: insights from In situ determination of benthic oxygen consumption. Geophysical Research Letters 45:27522760. 10.1002/2017gl076232

Mehrshad M, Rodriguez-Valera F, Amoozegar MA, Lopez-Garcia P, and Ghai R. 2017. The enigmatic SAR202 cluster up close: shedding light on a globally distributed dark ocean lineage involved in sulfur cycling. The ISME journal. 10.1038/s41396-017-0009-5

Nakatsuka T, Handa N, Harada N, Sugimoto T, and Imaizumi S. 1997. Origin and decomposition of sinking particulate organic matter in the deep water column inferred from the vertical distributions of its delta N-15, delta C13 and delta C-14. Deep-Sea Research Part I-Oceanographic Research Papers 44:1957-1979. 10.1016/s0967-0637(97)00051-4

Nogi Y, Hosoya S, Kato C, and Horikoshi K. 2004. Colwellia piezophila sp nov., a novel piezophilic species from deepsea sediments of the Japan Trench. International Journal of Systematic and Evolutionary Microbiology 54:1627-1631. 10.1099/ijs.0.03049-0

Nossa CW, Oberdorf WE, Yang L, Aas JA, Paster BJ, DeSantis TZ, Brodie EL, Malamud D, Poles MA, and Pei Z. 2010. Design of 165 rRNA gene primers for 454 pyrosequencing of the human foregut microbiome. World Journal of Gastroenterology 16:4135-4144. 10.3748/wjg.v16.i33.4135

Nunoura T, Nishizawa M, Hirai M, Shimamura S, Harnvoravongchai P, Koide O, Morono Y, Fukui T, Inagaki F, Miyazaki J, Takaki Y, and Takai K. 2018. Microbial diversity in sediments from the bottom of the Challenger Deep, the Mariana Trench. Microbes and Environments 33:186-194. 10.1264/jsme2.ME17194

Nunoura T, Takaki Y, Hirai M, Shimamura S, Makabe A, Koide O, Kikuchi T, Miyazaki J, Koba K, Yoshida N, Sunamura $\mathrm{M}$, and Takai K. 2015. Hadal biosphere: Insight into the microbial ecosystem in the deepest ocean on Earth. Proc Natl Acad Sci U S A 112:E1230-E1236. 10.1073/pnas.1421816112

Parks DH, Rinke C, Chuvochina M, Chaumeil P-A, Woodcroft BJ, Evans PN, Hugenholtz P, and Tyson GW. 2017. Recovery of nearly 8,000 metagenome-assembled genomes substantially expands the tree of life. Nature Microbiology 2:1533-1542. 10.1038/s41564-017-0012-7

Price MN, Dehal PS, and Arkin AP. 2009. FastTree: computing large minimum evolution trees with profiles instead of a distance matrix. Molecular Biology and Evolution 26:1641-1650. 10.1093/molbev/msp077

Rika E A, Mónica Torres B, Steven J H, and John A B. 2013. Microbial community structure across fluid gradients in the Juan de Fuca Ridge hydrothermal system. FEMS Microbiology Ecology 83:324-339.

Rinke C, Schwientek P, Sczyrba A, Ivanova NN, Anderson IJ, Cheng J-F, Darling A, Malfatti S, Swan BK, Gies EA, Dodsworth JA, Hedlund BP, Tsiamis G, Sievert SM, Liu W-T, Eisen JA, Hallam SJ, Kyrpides NC, Stepanauskas R, Rubin EM, Hugenholtz $P$, and Woyke T. 2013. Insights into the phylogeny and coding potential of microbial dark matter. Nature 499:431-437. 10.1038/nature12352

Schmid M, Walsh K, Webb R, Rijpstra WIC, van de Pas-Schoonen K, Verbruggen MJ, Hill T, Moffett B, Fuerst J, Schouten S, Damste JSS, Harris J, Shaw P, Jetten M, and Strous M. 2003. Candidatus "Scalindua brodae", sp nov., Candidatus "Scalindua wagneri", sp nov., two new species of anaerobic ammonium oxidizing bacteria. Systematic and Applied Microbiology 26:529-538. 10.1078/072320203770865837

Silvestro D, and Michalak I. 2012. raxmIGUI: a graphical front-end for RAxML. Organisms, Diversity \& Evolution 12:335-337. 10.1007/s13127-011-0056-0 
Taira K, Yanagimoto D, and Kitagawa S. 2005. Deep CTD casts in the Challenger Deep, Mariana Trench. Journal of Oceanography 61:447-454. 10.1007/s10872-005-0053-z

Tamburini C, Boutrif M, Garel M, Colwell RR, and Deming JW. 2013. Prokaryotic responses to hydrostatic pressure in the ocean - a review. Environmental Microbiology 15:1262-1274. 10.1111/1462-2920.12084

Tarn J, Peoples LM, Hardy K, Cameron J, and Bartlett DH. 2016. Identification of free-living and particle-associated microbial communities present in hadal regions of the Mariana Trench. Frontiers in Microbiology 7:665. 10.3389/fmicb.2016.00665

Tas N, van Eekert MHA, de Vos WM, and Smidt H. 2010. The little bacteria that can diversity, genomics and ecophysiology of 'Dehalococcoides' spp. in contaminated environments. Microbial Biotechnology 3:389402. 10.1111/j.1751-7915.2009.00147.x

Tian J, Fan L, Liu H, Liu J, Li Y, Qin Q, Gong Z, Chen H, Sun Z, Zou L, Wang X, Xu H, Bartlett D, Wang M, Zhang Y-Z, Zhang $\mathrm{X}-\mathrm{H}$, and Zhang $\mathrm{CL}$. 2018. A nearly uniform distributional pattern of heterotrophic bacteria in the Mariana Trench interior. Deep-Sea Research Part I-Oceanographic Research Papers 142:116-126. 10.1016/j.dsr.2018.10.002

Turnewitsch R, Falahat S, Stehlikova J, Oguri K, Glud RN, Middelboe M, Kitazato H, Wenzhoefer F, Ando K, Fujio S, and Yanagimoto D. 2014. Recent sediment dynamics in hadal trenches: Evidence for the influence of higherfrequency (tidal, near-inertial) fluid dynamics. Deep-Sea Research Part I: Oceanographic Research Papers 90:125-138. 10.1016/j.dsr.2014.05.005

Vanhove S, Vermeeren H, and Vanreusel A. 2004. Meiofauna towards the South Sandwich Trench (750-6300m), focus on nematodes. Deep Sea Research Part II-Topical Studies in Oceanography 51:1665-1687. 10.1016/j.dsr2.2004.06.029

Wang Q, Garrity GM, Tiedje JM, and Cole JR. 2007. Naive Bayesian classifier for rapid assignment of rRNA sequences into the new bacterial taxonomy. Applied and Environmental Microbiology 73:5261-5267. 10.1128/aem.00062-07

Wang Y, Gao Z-M, Li J, He L-S, Cui G-J, Li W-L, Chen J, Xin Y-Z, Cai D-S, and Zhang A-Q. 2019a. Hadal water sampling by in situ microbial filtration and fixation (ISMIFF) apparatus. Deep Sea Research Part I: Oceanographic Research Papers 144:132-137. 10.1016/j.dsr.2019.01.009

Wang Y, Huang J-M, Cui G-J, Nunoura T, Takaki Y, Li W-L, Li J, Gao Z-M, Takai K, Zhang A-Q, and Stepanauskas R. 2019b. Genomics insights into ecotype formation of ammonia-oxidizing archaea in the deep ocean. Environmental Microbiology. 10.1111/1462-2920.14518

Wenzhofer F, Oguri K, Middelboe M, Turnewitsch R, Toyofuku T, Kitazato H, and Glud RN. 2016. Benthic carbon mineralization in hadal trenches: Assessment by in situ $\mathrm{O}_{2}$ microprofile measurements. Deep-Sea Research Part I-Oceanographic Research Papers 116:276-286. 10.1016/j.dsr.2016.08.013

Wessel P, and Smith WH. 1998. New, improved version of Generic Mapping Tools released. Eos, Transactions American Geophysical Union 79:579-579.

Woebken D, Lam P, Kuypers MMM, Naqvi SWA, Kartal B, Strous M, Jetten MSM, Fuchs BM, and Amann R. 2008. A microdiversity study of anammox bacteria reveals a novel Candidatus Scalindua phylotype in marine oxygen minimum zones. Environmental Microbiology 10:3106-3119. 10.1111/j.1462-2920.2008.01640.x

Xu Y, Nogi Y, Kato C, Liang ZY, Ruger HJ, De Kegel D, and Glansdorff N. 2003. Psychromonas profunda sp nov., a psychropiezophilic bacterium from deep Atlantic sediments. International Journal of Systematic and Evolutionary Microbiology 53:527-532. 10.1099/ijs.0.02227-0

Peer) reviewing PDF | (2019:02:34810:2:0:NEW 7 Apr 2019) 
Xu Z, Wang M, Wu W, Li Y, Liu Q, Han Y, Jiang Y, Shao H, McMinn A, and Liu H. 2018. Vertical distribution of microbial eukaryotes from surface to the hadal zone of the Mariana Trench. Frontiers in Microbiology 9. 10.3389/fmicb.2018.02023

Zakrzewski M, Goesmann A, Jaenicke S, Juenemann S, Eikmeyer F, Szczepanowski R, Abu Al-Soud W, Sorensen S, Puehler A, and Schlueter A. 2012. Profiling of the metabolically active community from a production-scale biogas plant by means of high-throughput metatranscriptome sequencing. Journal of Biotechnology 158:248-258. 10.1016/j.jbiotec.2012.01.020

Zhang X, Xu W, Liu Y, Cai M, Luo Z, and Li M. 2018. Metagenomics reveals microbial diversity and metabolic potentials of seawater and surface sediment from a hadal biosphere at the Yap Trench. Frontiers in Microbiology 9. 10.3389/fmicb.2018.02402

\section{Figure legends:}

Fig. 1. Sampling sites in the Challenger Deep.

(A) The map was created with Generic Mapping Tools (GMT) (Wessel \& Smith 1998). The sampling sites from Challenger Deep were within a red frame in the map. (B) The sediment (yellow diamond) samples were collected during three cruises (R/V TS01, TS03 and DY37II). The sediment samples (water depth $>5,000 \mathrm{~m}$ ) were collected by manned submersible, hadal lander and boxcore sampler. Details for the samples were listed in Table S1.

Fig. 2. Relative abundance of prokaryotic phyla in the sediments from the Challenger Deep. The microbial communities were revealed based on sequencing of 16S rRNA gene amplicons and classifcation at the phylum level using the RDP classifier against the SILVA 132 database. The core lengths were indicated in the bracketes behind the sample ID.

Fig. 3. Principal Coordinates Analysis $(\mathrm{PCoA})$ and hierarchical clustering of the samples.

(A) PCoA of the microbial communities. The percentages of genera in the communities were used for calculation of Bray-Curtis dissimilarities and then a PCoA plot. Color code indicates the different water depths of the samples while different symbols distinguish the samples from each other (simple IDs refer to Table S3). (B) Hierarchical clustering of the samples was based on the Bray-Curtis dissimilarities. The samples from the trench-axis of the Challenger Deep were framed and labeled. 
551 Fig. 4. Phylogenetic trees for representative reads of 16S rRNA OTUs.

552 The bootstrap supports were based on 1000 replicates. The OTUs were most abundant in the 553 microbial communities. Their representative reads were collected to construct an ML tree (A for 554 archaea; B and C for Terrabacteria and FCB superphylum, respectively). 
Figure 1

Sampling sites in the Challenger Deep.

(A) The map was created with Generic Mapping Tools (GMT) (Wessel \& Smith 1998). The sampling sites from Challenger Deep were within a red frame in the map. (B) The sediment (yellow diamond) samples were collected during three cruises (R/V TS01, TS03 and DY37II). The sediment samples (water depth $>5,000 \mathrm{~m}$ ) were collected by manned submersible, hadal lander and box-core sampler. Details for the samples were listed in Table S1.

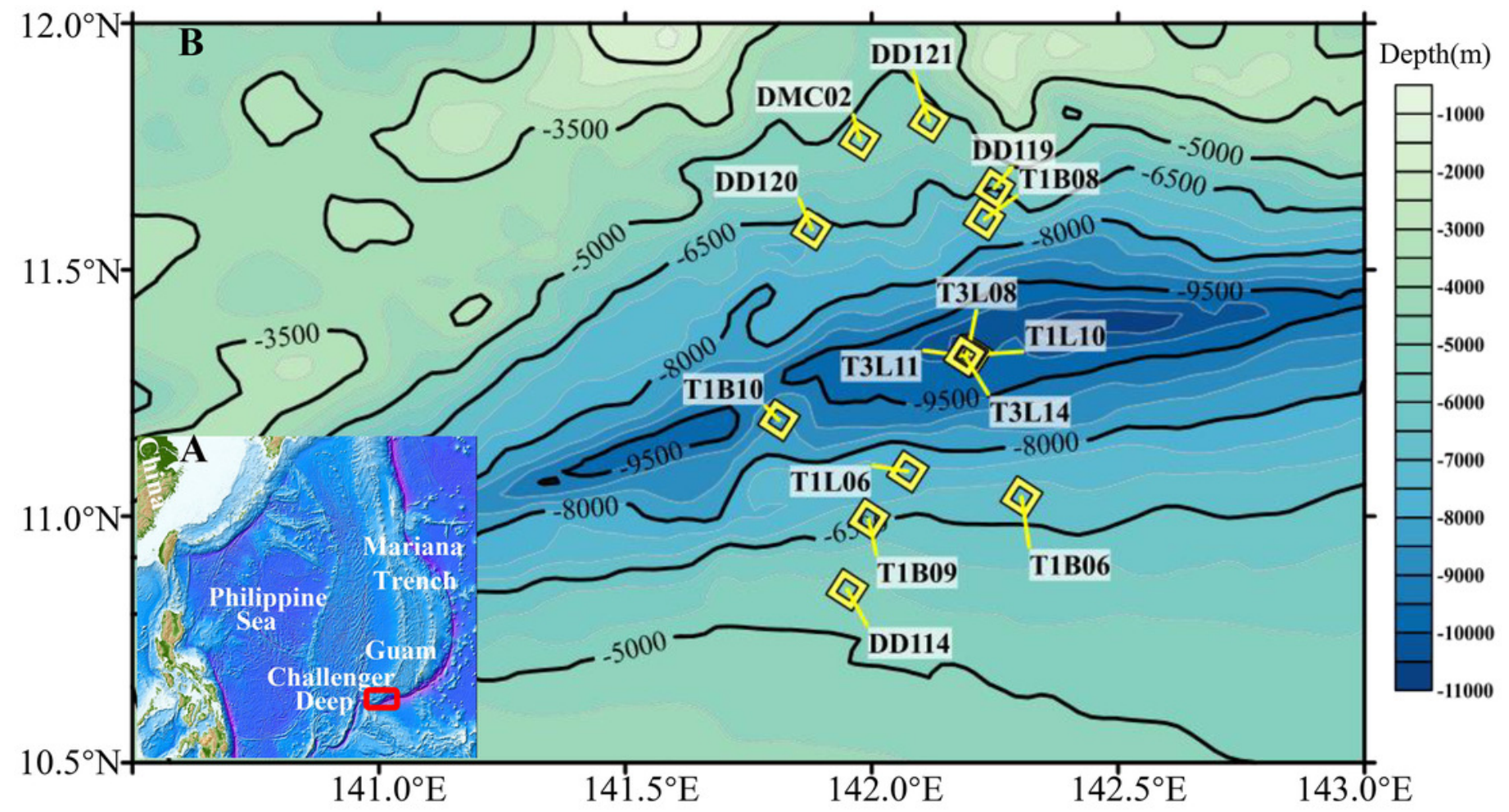




\section{Figure 2}

Relative abundance of prokaryotic phyla in the sediments from the Challenger Deep.

The microbial communities were revealed based on sequencing of 16S rRNA gene amplicons and classifcation at the phylum level using the RDP classifier against the SILVA 132 database. The core lengths were indicated in the bracketes behind the sample ID. 


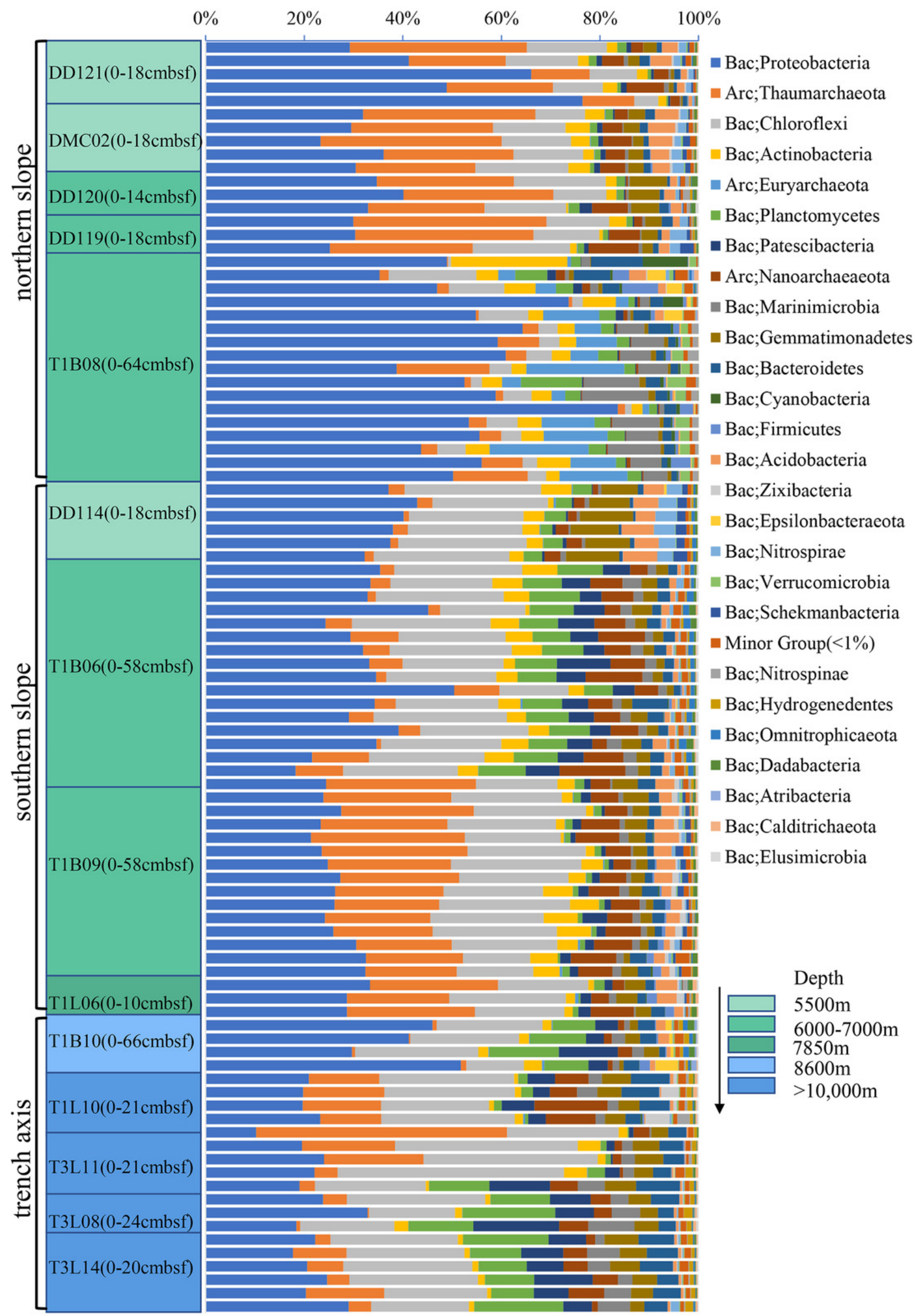




\section{Figure 3}

Principal Coordinates Analysis (PCoA) and hierarchical clustering of the samples.

(A) PCoA of the microbial communities. The percentages of genera in the communities were used for calculation of Bray-Curtis dissimilarities and then a PCoA plot. Color code indicates the different water depths of the samples while different symbols distinguish the samples from each other (simple IDs refer to Table S3). (B) Hierarchical clustering of the samples was based on the Bray-Curtis dissimilarities. The samples from the trench-axis of the Challenger Deep were framed and labeled. 

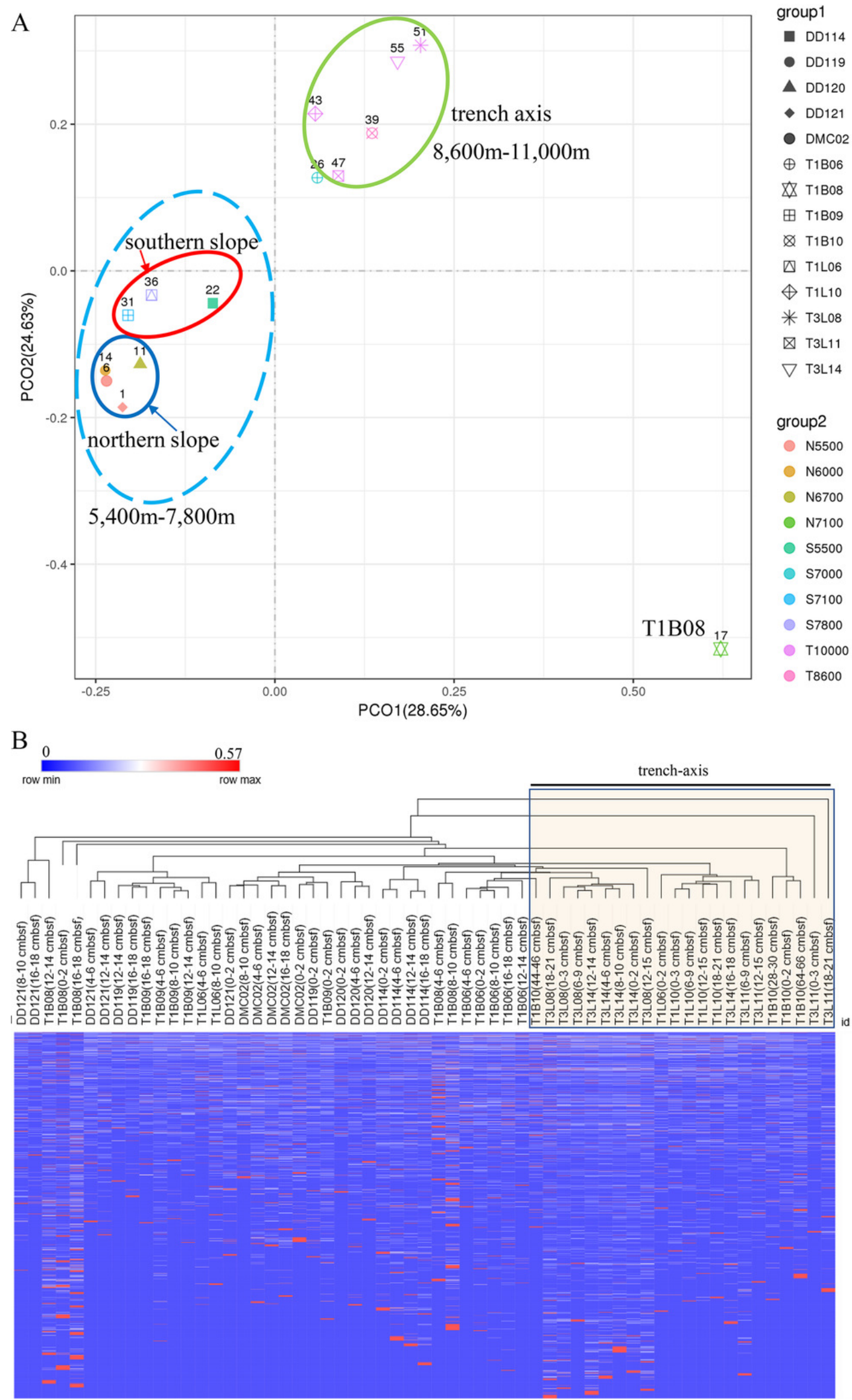
Figure 4

Phylogenetic trees for representative reads of $16 \mathrm{~S}$ rRNA OTUs.

The bootstrap supports were based on 1000 replicates. The OTUs were most abundant in the microbial communities. Their representative reads were collected to construct an ML tree (A for archaea; B and C for Terrabacteria and FCB superphylum, respectively). 


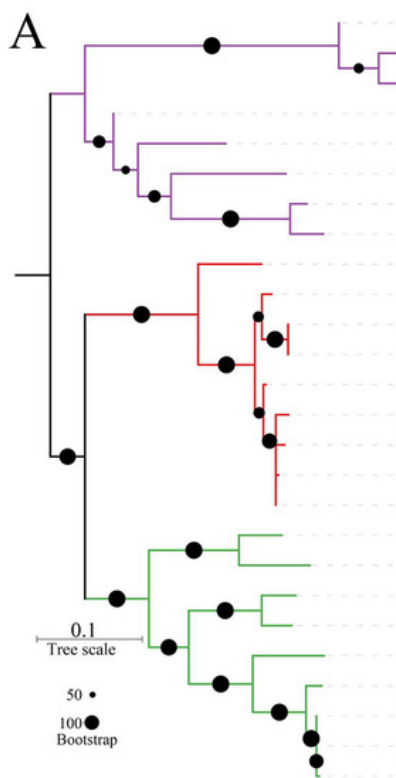

OTU146832 from Challenger Deep 10909m Woesearchaeota AR13 (JWKU01000011)

OTU81784 from Challenger Deep 5481 m OTU54927 from Challenger Deep 10909m Woesearchaeota AR11 (JWKT01000043)

Woesearchaeota AR20 (CP010426)

Woesearchaeota marine saline water (CEPX01003170)

OTU227271 from Challenger Deep 10909m Nitrososphaera viennensis strain EN76 (NR_134097) OTU88520 from Challenger Deep 6464m abyssal sediment from South Pacific 5076m (FJ487503) OTU340637 from Challenger Deep 6499m Nitrosopumilus maritimus SCM1 (CP000866) water from Puerto Rico Trench 6000m (HM799353) cold-seep sediments from Japan Trench (AB189387) sediments from Ogasawara Trench (AB583377) OTU214093 from Challenger Deep 10908m

Thermoplasma volcanium (NC_002689)

Ferroplasma acidarmanus (NC_021592)

water from Mediterranean Sea 450m (AF223150)

water from Antarctic Polar Front 3000m (AF257278)

water from Antarctic Polar Front 3000m (AF257277)

water from Antarctic Polar Front 500m (AY534910)

water from North Pacific Subtropical Gyre 500m (DQ156457)

OTU100704 from Challenger Deep 7143m

hydrothermal plume from Juan de Fuca Ridge (JQ678181)

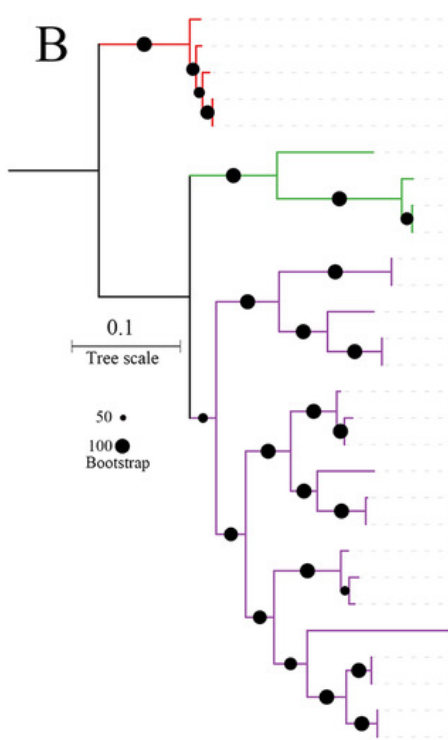

Cyanobium gracile PCC 6307 (NR 114406)

OTU231835 from Challenger Deep 10909m

Prochlorococcus MIT9313 (NC_005071)

Synechococcus CC9902 (NC_007513)

OTU61581 from Challenger Deep 7143m

Euzebya tangerina strain F10 (NR_112876)

water from Sargasso Sea 80m (AF110142)

Scleractinia from La Parguera (JQ515472)

OTU338607 from Challenger Deep 7022m

sediment from South Pacific (LC081115)

OTU330041 from Challenger Deep $10911 \mathrm{~m}$

Thermomarinilinea lacunifontana strain SW7 (NR_132293)

OTU24314 from Challenger Deep 10909m

sediment from Shimokita Penninsula (AB806012)

seafloor from Southern Mariana Trough 3000m (AB858598)

OTU344095 from Challenger Deep 10953m

sediment from South Pacific 5306m (FJ746197)

Dehalogenimonas formicexedens strain NSZ-14 (NR 156917)

Dehalococcoides ethenogenes (AF004928)

Dehalococcoides mccartyi strain 195 (NR_074116)

OTU87119 from Challenger Deep 10909m

sediment from South Pacific (HQ721372)

OTU22050 from Challenger Deep 10911m

OTU124775 from Challenger Deep $7121 \mathrm{~m}$

sediments from in the Challenger Deep 500m (LC050009)

OTU335610 from Challenger Deep $10911 \mathrm{~m}$

seawater from South Pacific Gyre (HQ721461)

OTU161067 from Challenger Deep $10911 \mathrm{~m}$

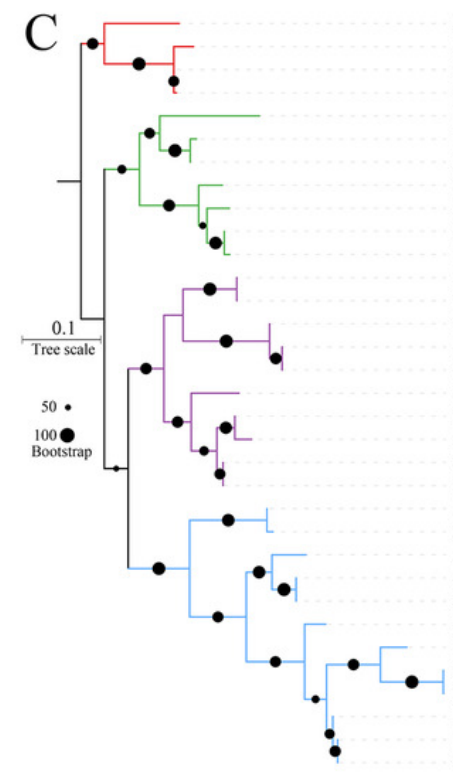

Zixibacteria (AUYT00000000)

sediment from South Pacific (LC081048)

sediments from Mariana subduction (MG580245)

Gemmatirosa kalamazoonesis strain KBS708 (NR 132675)

hydrothermal field from East Lau 2350m (FJ205250)

OTU138687 from Challenger Deep 10908m

Gemmatimonadetes AAA240-J22 (GCA 000482625)

OTU386217 from Challenger Deep $10909 \mathrm{~m}$

marine sponge from North Atlantic (KF597116)

marine sponge from North Atlantic (KF5971

OTU247321 from Challenger Deep $10911 \mathrm{~m}$

Marinimicrobia AAA003-E22 (ASPI01000040)

OTU384236 from Challenger Deep $10911 \mathrm{~m}$

marine sponge from North Atlantic (HQ721397)

OT 383320 rom Challenger Deep $10908 \mathrm{~m}$

OTU147865 from Challenger Deep 7143m

OTU50751 from Challenger Deep 7143m

Marinimicrobia 0000113-D11 (AYLG01000001)

OTU237409 from Challenger Deep 10908m

OTU333961 from Challenger Deep 10909m

OTU34232 from Challenger Deep $7022 \mathrm{~m}$

sponge from Red Sea (JQ062842)

Fabivirga thermotolerans strain A-4 (NR 148597)

OTU251565 from Challenger Deep $1090 \overline{8} \mathrm{~m}$

OTU110732 from Challenger Deep $10953 \mathrm{~m}$

Flavimarina pacifica strain IDSW-73 (NR 148260)

Flavobacterium aquicola strain TMd3a3 (NR_151881)

OTU135606 from Challenger Deep $10911 \mathrm{~m}$

sponge from Red Sea (JQ062610)

Leeuwenhoekiella nanhaiensis strain G18 (NR_144589)

water from Mediterranean Sea (DQ294291 